\title{
Soluble Tei2 fusion protein inhibits retinopathy of prematurity occurrence via regulation of the Ang/Tie2 pathway
}

\author{
WEIJING LI*, WEIHUA ZHANG ${ }^{*}$, CUIYING ZHANG, \\ CHUNFANG ZHU, XIANGLING YI, YAN ZHOU and YAN LV
}

Department of Ophthalmology, Liaocheng People's Hospital, Liaocheng, Shandong 252000, P.R. China

Received August 14, 2018; Accepted March 23, 2019

DOI: $10.3892 /$ etm.2019.7608

\begin{abstract}
The aim of the present study was to investigate the potential mechanism of retinopathy of prematurity (ROP) using an oxygen-induced retinopathy (OIR) mouse model. For experiments, mice were divided into either the OIR group or control group. Fluorescein isothiocyanate-dextran cardiac perfusion and stretched retina preparation were performed. The total retina area, area of instillation, density of microvascular network, area of new blood vessels, vein width and the tortuosity of arteries were measured. Next, mice were randomly assigned into the PBS, soluble TEK receptor tyrosine kinase (sTie2)-fusion protein (Fc), angiopoietin 1 (Ang1), ranibizumab, ranibizumab + sTie2-Fc and ranibizumab + Ang1 treatment groups. Following housing for 5 days, the body weight of each mouse was recorded. Mice in the OIR group presented smaller total retina area and larger area of instillation, larger area of new blood vessels, and higher microvascular network density compared with the control PBS group. Obvious retinal vein dilatation and arterial tortuosity were identified in the OIR group. The amount of endotheliocyte nuclei of new vessels beyond the inner limiting membrane was larger in the OIR group compared with the control group. Furthermore in the next set of experiments, a larger area of instillation, smaller area of new blood vessels and decreased amount of endotheliocyte nuclei of new vessels were observed in the sTie2-Fc group, Ang1 group, ranibizumab group, ranibizumab + sTie2-Fc group and ranibizumab + Ang1 group compared with the PBS group. Specifically, the ranibizumab + sTie2-Fc group and ranibizumab + Ang1 group demonstrated markedly reduced retina instillation area and microvascular network density
\end{abstract}

Correspondence to: Dr Yan Lv, Department of Ophthalmology, Liaocheng People's Hospital, 67 Dongchang West Road, Dongchangfu, Liaocheng, Shandong 252000, P.R. China

E-mail: yankelvyan@126.com

*Contributed equally

Key words: oxygen-induced retinopathy, soluble TEK receptor tyrosine kinase fusion protein, angiopoietin/TEK receptor tyrosine kinase, retinopathy of prematurity in the instillation area. Total retina area and body weight following 10 days of the experiment for the ranibizumab group were significantly lower compared with other groups. In conclusion, the combined regulation of the Ang/Tie2 and the vascular endothelial growth factor (VEGF)/VEGF receptor pathways markedly increased the efficacy of treatment with retinal neovascularization (RNV). Regulation of these pathways has a potential for treating RNV, in particular ROP.

\section{Introduction}

Neovascular (NV) ophthalmopathy includes proliferative diabetic retinopathy, age-related macular degeneration, and retinopathy of prematurity (ROP), which have become the main causes of blindness in the elderly (1), infants and young children (2).

The development of NV ophthalmopathy consists of two stages. First, vascular occlusion and disordered blood vessels develop from existing conditions. Subsequently, various hypoxia-related growth factors, including vascular endothelial growth factor (VEGF), angiopoietin (Ang)-2 and erythropoietin, are produced due to the insufficient blood and oxygen supply. These factors further lead to angiogenic lesions (3-6). Animal models of oxygen-induced retinopathy (OIR) have been frequently used to study the mechanism of ROP (7-9). Hence, the present study established an OIR mouse model to explore the potential mechanism of NV ophthalmopathy.

Retinal neovascularization (RNV) treatments include retinal laser photocoagulation and vitreous surgery. However, these treatments were only capable of reducing retinal oxygen consumption, vitreous proliferation and traction, while the retina blood supply was not improved or restored to the normal level. Currently, various anti-VEGF drugs are being utilized in ophthalmology due to the research into VEGF and its corresponding receptors. However, anti-VEGF drugs have limitations, for example, anti-VEGF therapy can transiently reduce systemic VEGF levels (10). In addition, the formation and traction of preretinal proliferative membranes was accelerated following anti-VEGF administration, further worsening the condition $(11,12)$. Anti-VEGF drugs have a poor therapeutic effect on macular edema due to a short effective duration and a high rate of relapse (13-15). Notably, anti-VEGF therapy for ROP inhibits normal structural development and functional maturation of retinal nerve cells. It is also reported 
that anti-VEGF treatment affects development of multiple organs, in particular the lungs (16). Therefore, a safer and more effective therapy for ROP is urgently required.

Ang and its receptor TEK receptor ty rosine kinase 2 (Tie2) have been recently identified to serve a role in the angiogenic pathway (17-24). Ang1 and Ang2 regulate the stability of endothelial cells by binding to their coreceptor Tie2. This indicates that Ang1 can enhance the adhesion of vascular peripheral tissues and stabilize newly formed blood vessels, thereby limiting persistent abnormal angiogenesis (23). Ang2 antagonizes the effects of Ang1 and enhances the sensitivity of endothelial cells to mitogenic signals, leading to instability of endothelial cells and promoting immature neovascularization (23). Therefore, it is generally hypothesized that Ang2 inhibition or Ang1 promotion can inhibit the formation of new blood vessels. The Ang/Tie2 pathway mainly affects vascular structure and maturation without significantly overlapping with the VEGF/VEGF receptor (R) pathway spectrum.

Ranibizumab is a recombinant human monoclonal antibody that binds to all high-affinity VEGFs and blocks the corresponding VEGF/VEGFR pathway (25). Soluble Tie2 fusion protein (sTie-Fc) is the extracellular component of Tie2, which competitively binds to Ang1 and Ang2, thus regulating the Tie2 pathway in endothelial cells (26-30).

The present study established a mouse model of OIR to compare the effects of the VEGF/VEGFR and Ang/Tie2 pathways on regulating RNV to provide novel directions for RNV treatment.

\section{Materials and methods}

Experimental animals. A total of 80 male C57BL/6J mice (7-days-old) were obtained from Shanghai SLAC Laboratory Animal Co., Ltd. Mice were housed in a temperature $\left(21 \pm 2^{\circ} \mathrm{C}\right)$ and humidity (40-70\%) controlled room under a $12 \mathrm{~h}$ light/dark cycle (lights on at 06:00). Mice were maintained in an experimental animal center under specific-pathogen-free conditions, and were given free access to water and food. This study was approved by the Animal Ethics Committee of Liaocheng People's Hospital Animal Center.

Model and treatment. For the OIR model group, 14 C57BL/6J mice (age, 7 days) were housed in a $75 \%$ oxygen concentration hyperoxia container for 5 consecutive days, followed by 5 days in a normal air environment. For the control group, $10 \mathrm{C} 57 \mathrm{BL} / 6 \mathrm{~J}$ mice (age, 7 days) were housed in a normal air environment for 10 days. In addition, 120 C57BL/6J mice (age, 7 days) were selected and divided into 6 groups $(n=20)$. The mice were kept in a container with $75 \%$ oxygen concentration for 5 consecutive days. At 12-days old, the weight of the mice was measured and recorded. PBS $(1 \mu \mathrm{l}), 1 \mu \mathrm{l}$ sTie2-Fc (3 mol/l; cat. no. SFC-014; Ankang Biotechnology Co., Ltd.), $1 \mu \mathrm{l}$ Angl (4 mol/l; cat. no. An-002; Ankang Biotechnology Co., Ltd.), $1 \mu \mathrm{l}$ ranibizumab (10 mol/l; Shanghai TheraMabs Bio-technology Co., Ltd.), $1 \mu 1$ sTie2-Fc $+1 \mu 1$ ranibizumab (sTie2-Fc $3 \mathrm{~mol} / \mathrm{l}$; ranibizumab $10 \mathrm{~mol} / \mathrm{l}$ ) or $1 \mu \mathrm{l} \mathrm{Angl}+1 \mu \mathrm{l}$ ranibizumab (Angl $4 \mathrm{~mol} / \mathrm{l}$; ranibizumab $10 \mathrm{~mol} / \mathrm{l}$ ) were injected into the vitreous cavity of mice. Mice were reared for further 5 days then the weight of the mice was again measured and recorded at 10 days. Half of the mice were subsequently sacrificed at 5 days and the remainder were sacrificed at 10 days.

Fluorescein isothiocyanate (FITC)-dextran cardiac perfusion and stretched retina preparation. A total of 10 mice were anesthetized by intraperitoneal injection of ketamine $(120 \mathrm{mg} / \mathrm{kg})$ and xylazine $(12 \mathrm{mg} / \mathrm{kg})$, as previously descried (31-33). The heart was exposed and $1 \mathrm{ml}$ of FITC-dextran (2\%; cat. no. 141270; Shanghai Huicheng Biotechnology Co., Ltd.) was administrated into the apex of left ventricle. After 3-5 min, pupils were harvested then the mucous membranes stained and fixed in $4 \%$ paraformaldehyde at room temperature for 20-30 $\mathrm{min}$. The retinas were stripped from the lens and cut into $6 \mu \mathrm{m}$ sections. The total retina area, area of instillation, microvascular network density, area of new blood vessels, width of vein and the tortuosity of the arteries were observed.

Quantification of endotheliocyte nuclei of new vessels beyond the inner limiting membrane. A total of 5 mouse pupils from each group harvested as aforementioned were collected and fixed in $4 \%$ paraformaldehyde at room temperature for $24 \mathrm{~h}$. The lens was removed under a microscope, then subjected to a gradient dehydration in alcohol $(100,95,85$ and $75 \%)$ and xylene. Subsequently, tissue samples were paraffin embedded and cut into $5 \mu \mathrm{m}$ sections for hematoxylin $(5 \mathrm{~min})$ and eosin (1-3 min; HE) staining (Boster Biological Technology; 5 min). The total number of endotheliocyte nuclei of new vessels beyond the inner limiting membrane were counted in each sample via light microscopy. Three retinal tissue sections were analyzed for each mouse and three fields of view were analyzed for each sample. The total number of endotheliocyte nuclei was taken as an average from three technicians who were blinded to the study groups.

Intravitreal injection. Following anesthesia by intraperitoneal injection of ketamine $(120 \mathrm{mg} / \mathrm{kg})$ and xylazine $(12 \mathrm{mg} / \mathrm{kg})$, 30 mice were sacrificed at day 10 and their eyelids were separated with ophthalmic smooth forceps. Pupils were immediately dilated with tropicamide $(0.01 \%$; Sigma-Aldrich; Merck KGaA; cat. no. T9778) 2-4 times. The eyeball was protruded by slight pressure on the orbital margin then $1 \mu \mathrm{l}$ sTie2-Fc (3 mol/l), Angl (4 mol/l), ranibizumab (10 mol/l), sTie2-Fc + ranibizumab (sTie2-Fc $3 \mathrm{~mol} / \mathrm{l}$; ranibizumab $10 \mathrm{~mol} / \mathrm{l}$ ) or Angl + ranibizumab (Angl $4 \mathrm{~mol} / \mathrm{l}$; ranibizumab $10 \mathrm{~mol} / \mathrm{l}$ ) were administrated from the superior corneal limbus to the vitreous chamber. The needle of microinjector was maintained in the chamber for $15 \mathrm{sec}$ and immediately pulled out. After intravitreal injection, erythromycin ophthalmic ointment was applied.

Statistical analysis. Stata statistical software (version 7.0; StataCorp LP) was used for data analysis. Data are presented as the mean \pm standard deviation. The differences amongst groups were compared using one-way analysis of variance, followed by Fisher's least significant difference post hoc test with Bonferroni adjustment for comparisons between two groups. $\mathrm{P}<0.05$ was considered to indicate a statistically significant difference. 
A

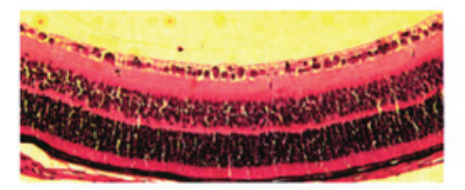

B

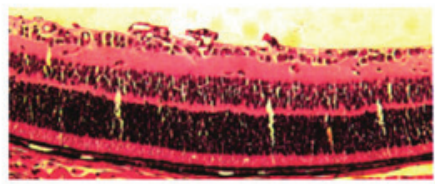

C

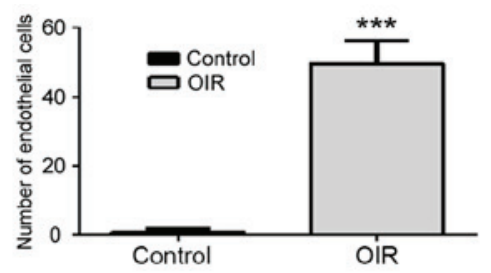

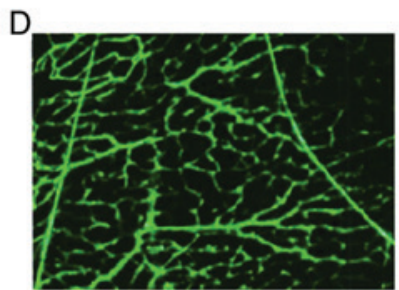
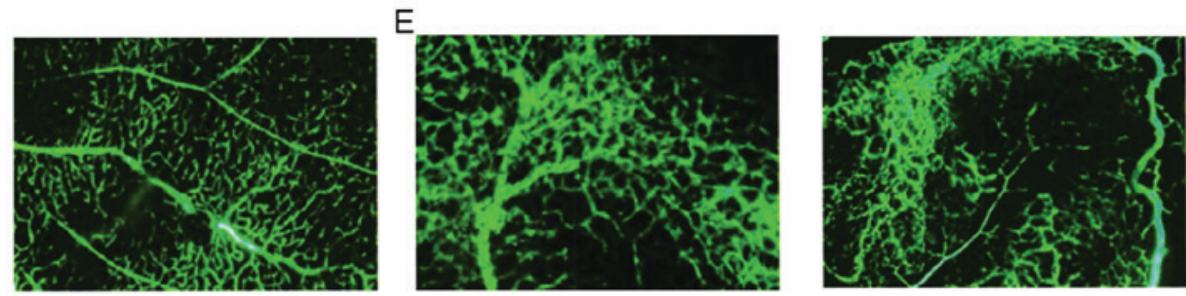

Figure 1. Pathological lesions in mouse pupils for the OIR and control groups. (A) HE staining of mouse pupils in the control group. (B) HE staining of mouse pupils in the OIR group demonstrating a large number of endotheliocyte nuclei of new vessels beyond the inner limiting membrane (magnification, x200). (C) Quantification of the number of endothelial cells in the OIR group and control group. (D) Pathological mouse retina changes in the control group. (E) Pathological mouse retina changes in the OIR group (magnification, $\mathrm{x} 50$ ). ${ }^{* * *} \mathrm{P}<0.001$ vs. control. OIR, oxygen-induced retinopathy; HE, hematoxylin and eosin.
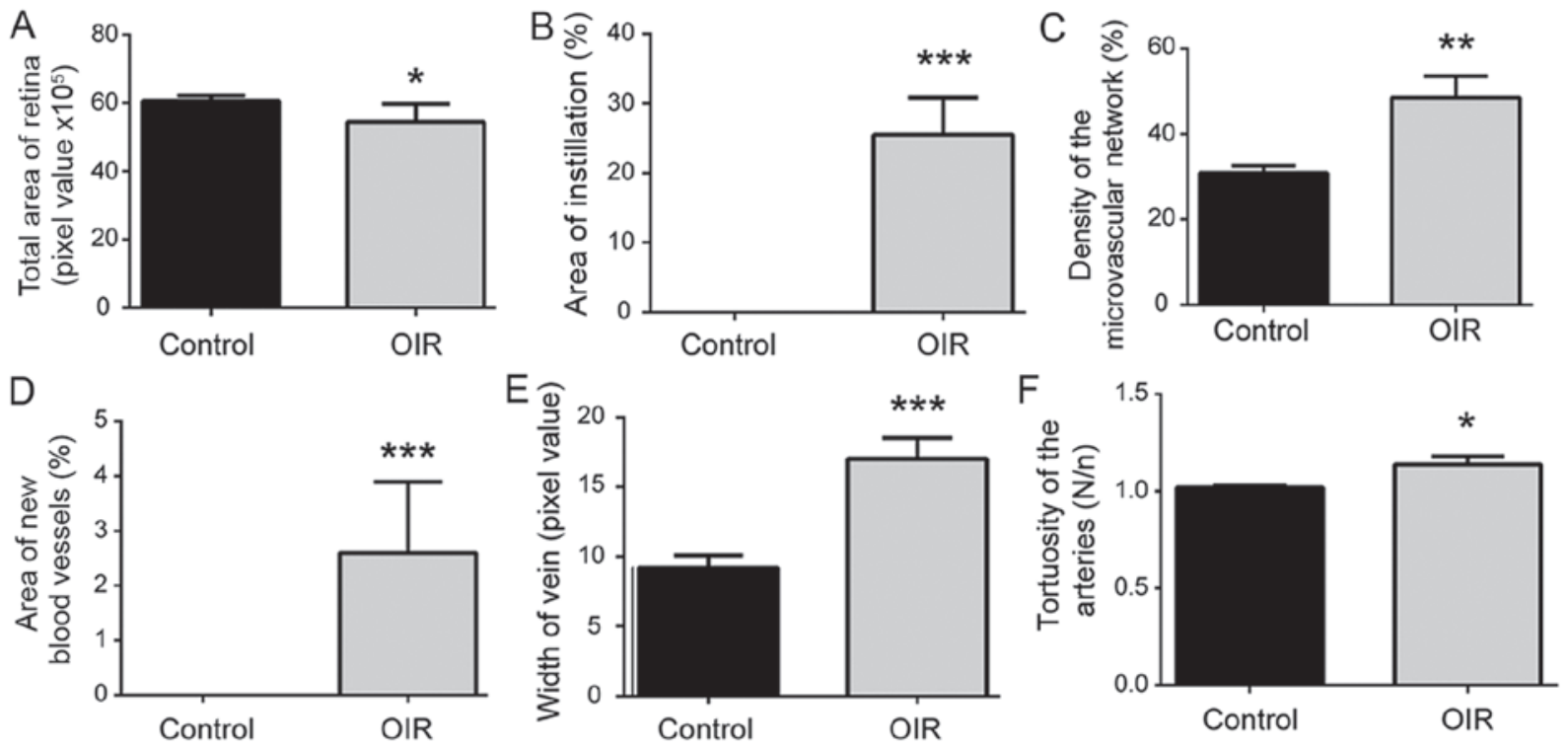

Figure 2. Comparison of retinal vascular development in the OIR group and control group. (A) Total area of retina. (B) Area of instillation. (C) Density of the microvascular network. (D) Area of new blood vessels. (E) Width of vein. (F) Tortuosity of the arteries. ${ }^{*} \mathrm{P}<0.05,^{* *} \mathrm{P}<0.01$ and ${ }^{* * *} \mathrm{P}<0.001$ vs. control. OIR, oxygen-induced retinopathy.

\section{Results}

Evaluation of retinal vascular development. The OIR model was constructed for evaluating the total amount of endotheliocyte nuclei of new vessels beyond the inner limiting membrane. Mice were randomly assigned into the OIR group $(n=10)$ or control group $(n=10)$. Results demonstrated that mice in the OIR group presented multiple neovascular clusters (Fig. 1A and B) and a significantly higher amount of endotheliocyte nuclei of new vessels compared with the control group (Fig. 1C). Retinal vein dilatation and arterial circuitous degree was used to assess the disease condition. Both parameters were more pronounced in the OIR group compared with the control group (Fig. 1D, E). Mice in the OIR group presented smaller total retina areas, a larger area of instillation, a larger area of new blood vessels, and a higher microvascular network density compared with the control PBS group. Marked retinal vein dilatation and arterial tortuosity were identified in the OIR group. The number of endotheliocyte nuclei of new vessels beyond the inner limiting membrane was larger in the OIR group compared with the control group. The results suggest that there was impaired retinal vascular development (Fig. 2).

Treatment with sTie2-Fc alleviates $R N V$ via regulating the Ang/Tie2 pathway. Mice received intravitreal injection of PBS, sTie2-Fc, Ang1, ranibizumab, ranibizumab + sTie2-Fc or ranibizumab + Ang1. Compared with the PBS group, mice injected with sTie2-Fc, Ang1 or ranibizumab presented less endotheliocyte nuclei of new vessels beyond the inner limiting membrane (Fig. 3A-F). The results demonstrated that individual administration of sTie2-Fc, Ang1 and ranibizumab did not affect retinal vascular development which 

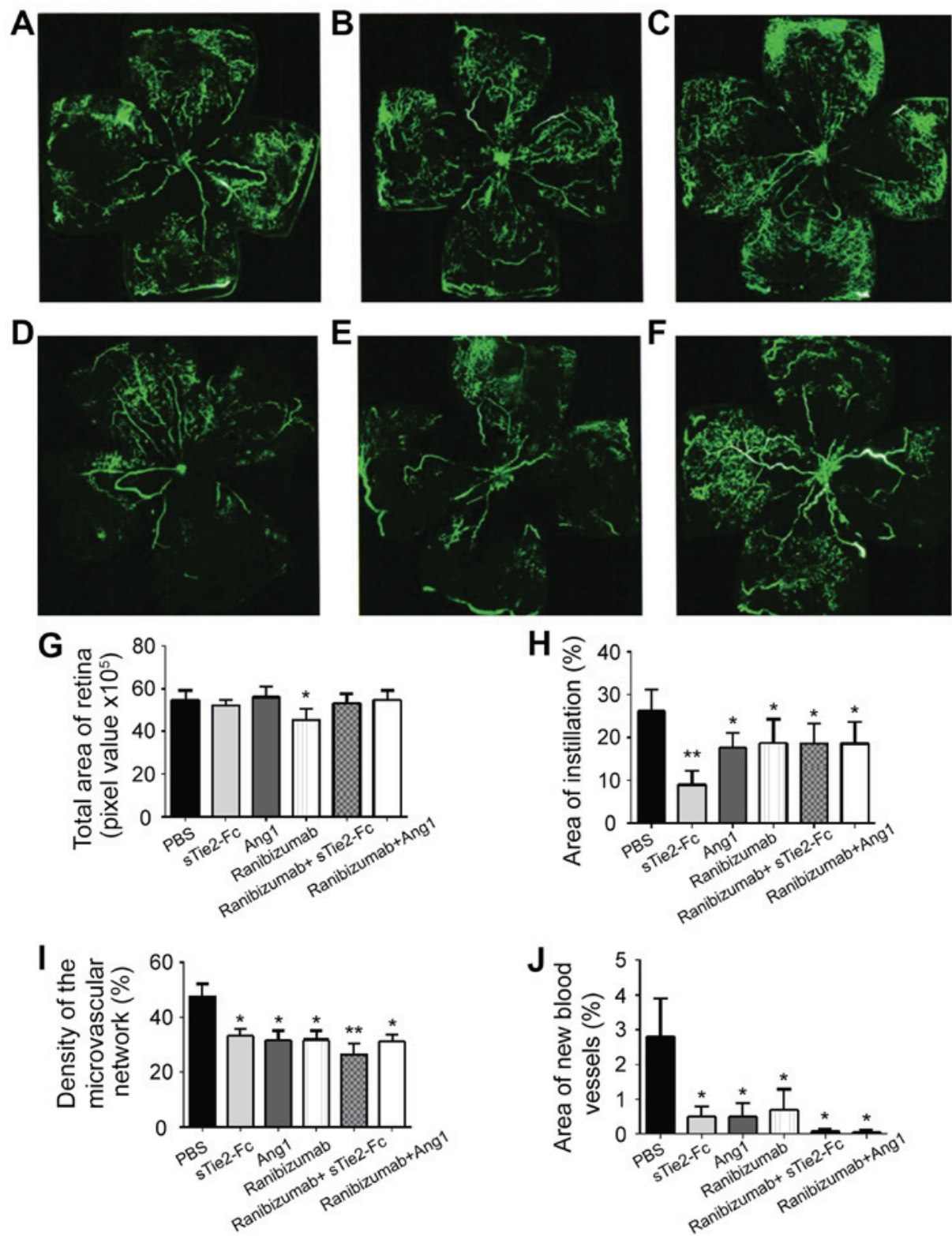

Figure 3. Treatment with sTie2-Fc alleviates retinal neovascularization via regulating the Ang/Tie2 pathway. Pathological changes of mouse retina in the (A) PBS, (B) sTie2-Fc, (C) Ang1, (D) ranibizumab, (E) ranibizumab + sTie2-Fc and (F) ranibizumab + Ang1 group (magnification, x50). (G) Total area of retina. (H) Area of instillation. (I) Density of the microvascular network. (J) Area of new blood vessels. ${ }^{*} \mathrm{P}<0.05$ and ${ }^{* *} \mathrm{P}<0.01$ vs. PBS group. sTie2-Fc, soluble TEK receptor tyrosine kinase-fusion protein; Ang1, angiopoietin1.

would manifest as a larger area of FITC-dextran instillation compared with PBS group (Fig. 3H). In addition, it was determined that sTie2-Fc administration may promote retinal vascular development compared with the PBS group (Fig. 3G-J) which suggested that sTie2-Fc may be a preferred option for RNV treatment. Ranibizumab + sTie2-Fc treatment markedly inhibited retinal vascular development, presenting as a smaller area of instillation and lower density of the microvascular network when compared with the PBS group (Fig. $3 \mathrm{H}$ and I). By contrast, ranibizumab + Angl administration did not affect retinal vascular development compared with the PBS group (Fig. 3G-J). Therefore ranibizumab + Angl treatment was considered the better option when compared with Ranibizumab + sTie2-Fc for treating RNV via the combined regulation of the Ang/Tie2 and VEGF/VEGFR pathways.
Combination of STie2-Fc or Angl with ranibizumab alleviates $R N V$. By comparing the HE staining amongst different groups, it was identified that intravitreal injection of sTie2-Fc, Ang1, ranibizumab, ranibizumab + sTie2-Fc and ranibizumab + Angl reduced the amount of endotheliocyte nuclei of new vessels beyond the inner limiting membrane compared with the PBS group. Ranibizumab + sTie2-Fc and ranibizumab + Ang1 treatment groups displayed a smaller quantity of endotheliocyte nuclei of new vessels beyond the inner limiting membrane compared with the sTie2-Fc, Ang1 and ranibizumab groups (Fig. 4). Combined administration of ranibizumab + sTie2-Fc and ranibizumab + Angl demonstrated better treatment efficacy than individual administration (Fig. 4G). However, no significant difference in the amount of endotheliocyte nuclei of new vessels beyond the inner limiting membrane was demonstrated between ranibizumab + sTie2-Fc and ranibizumab + Angl 


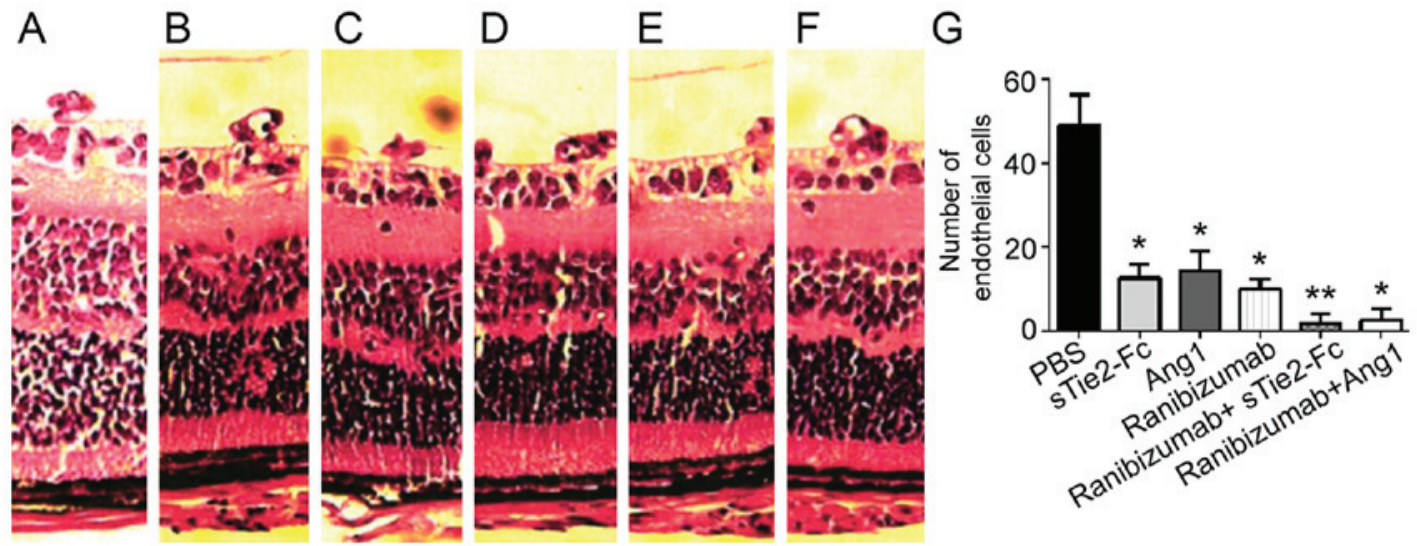

Figure 4. Combination treatment of sTie2-Fc or Ang1 with ranibizumab alleviates retinal neovascularization. Hematoxylin and eosin staining of mouse pupils in the (A) PBS group, (B) sTie2-Fc group, (C) Ang1 group, (D) ranibizumab group, (E) ranibizumab + sTie2-Fc group and (F) ranibizumab + Ang1 group (magnification, $\mathrm{x} 200$ ). (G) Number of endothelial cells in the different groups. $\mathrm{P}<0.05$ and ${ }^{* *} \mathrm{P}<0.01$ vs. PBS group. sTie2-Fc, soluble TEK receptor tyrosine kinase-fusion protein; Ang1, angiopoietin1.
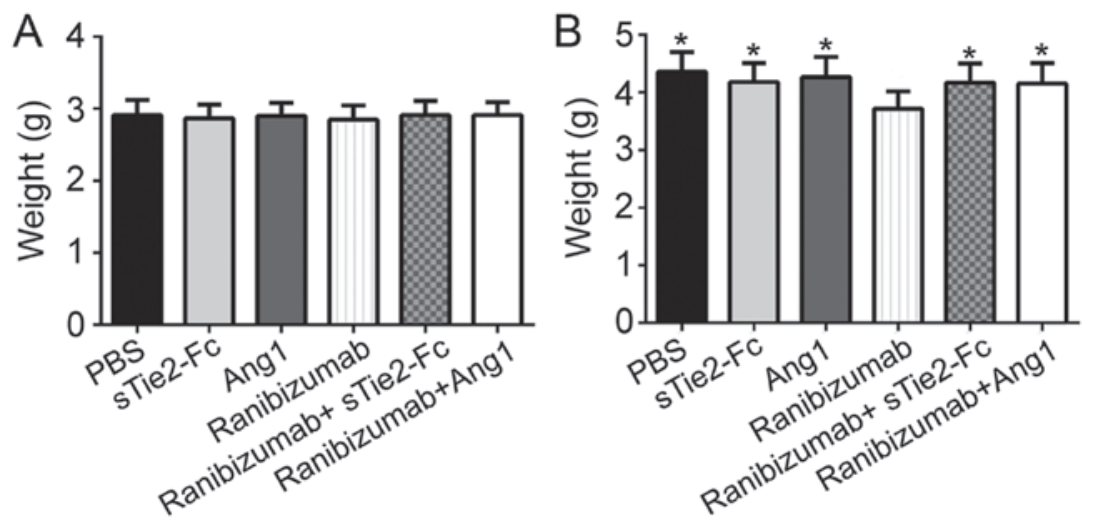

Figure 5. Ranibizumab affects the normal development of mice with oxygen-induced retinopathy. Body weight of mice in different groups following (A) 5 days and (B) 10 days of experiments. "P<0.05 vs. the ranibizumab group. sTie2-Fc, soluble TEK receptor tyrosine kinase-fusion protein; Ang1, angiopoietin1.

groups. These results indicated that a combination of sTie2-Fc or Ang1 with ranibizumab alleviated RNV.

Ranibizumab affects normal development of OIR mice. Subsequently, the potential mechanism of ranibizumab in regulating RNV development was investigated. Results demonstrated that there was no significant difference in mouse weight between any groups following 5 days of experimentation (Fig. 5A). Treatment with sTie2-Fc or Ang1 + ranibizumab had no effect on body weight following 5 days of experimentation. However, body weight was significantly decreased in the ranibizumab group compared with the other groups following 10 days of experimentation (Fig. 5B). This result suggested that ranibizumab may affect the normal development of OIR mice.

\section{Discussion}

The OIR animal model simulates RNV symptoms, in particular ROP, and therefore has been commonly utilized to investigate the underlying mechanism of ROP (7-9). RNV development is considered to be the result of the imbalance between local angiogenesis promoting and inhibitory factors $(23,24)$. VEGF and VEGFR are considered to be the most important angiogenesis promoting factors $(4,5)$. Intravitreal injections of various VEGF antagonists are used for anti-neovascular therapy (34-36). Ranibizumab is a recombinant human monoclonal antibody that binds to all isoforms of VEGF with high affinity, does not contain a fragment crystallizable region and inhibits the VEGF/VEGFR signaling pathway (25). Ranibizumab received Food and Drug Administration certification in 2006 and is currently approved for clinical ophthalmology in China (25).

Previous studies have identified severe limitations of anti-VEGF therapy for ROP (10-12); therefore, research has focused on the development of more effective medication for this condition. In recent years, the Ang/Tie2 pathway has been identified to inhibit angiogenesis (26-30). Ang1 administration inhibits angiogenesis by enhancing the interaction between endothelial cells and pericytes (37,38). The Ang/Tie2 pathway mainly affects vascular plasticity and maturation. In Ang2 heterozygous knockout mice, it was determined that Ang2 modulates hypoxia-induced neovascularization by regulating MMP activity (39).

In the present study, an OIR mouse model was first established. Then mice received intravitreal injection of PBS, sTie2-Fc, Ang1, ranibizumab, ranibizumab + sTie2-Fc or ranibizumab + Ang1. Results demonstrated that individual 
administration of Ang1 and ranibizumab both markedly alleviated RNV without affecting retinal vascular development. Intravitreal injection of sTie2-Fc promoted retinal vascular development, manifesting as a smaller instillation retina area compared with the PBS group. Therefore, it was hypothesized that sTie2-Fc may be a preferred option compared with ranibizumab for treating RNV.

Compared with individual administration of sTie2-Fc, ranibizumab or Ang1, the inhibitory effect of ranibizumab + sTie2-Fc and ranibizumab + Ang1 on RNV was more pronounced. The present results indicated that a combination treatment targeting the Ang/Tie 2 and VEGF/VEGFR pathways could better alleviate RNV. Takagi et al (29) demonstrated that the combined inhibition of Tie 2 and VEGF signaling may be effective in preventing pathologic angiogenesis in ischemic retinal disorders, which is in agreement with our results.

In particular, the present study identified that mice injected with ranibizumab demonstrated a decreased total retina area and lower body weight. In clinical research, baby weight gain is closely related to the development of multiple organs, especially the lungs $(40,41)$. Anti-VEGF therapy has been reported to inhibit multiple organ development in infants (16). Changes in blood VEGF levels have not been observed following treatment with ranibizumab (42), therefore the inhibition of organ development may not be associated with ranibizumab. Further studies on the inhibitory effect of ranibizumab are required to fully elucidate the potential side effects. The present study demonstrated the changes in RNV on day 12 based on the preliminary experimental results; however, future experiments should investigate the degree of neovascularization beyond 12 days in the different experimental groups. In addition, the investigation into the role of Ang2 in RNV using Ang2 inhibitors should be performed.

In conclusion, sTie2-Fc alleviated RNV without affecting retinal vascular development by activating the Ang/Tie2 pathway. It was also determined that intravitreal administration of ranibizumab may inhibit normal growth and development of mice. Regulation of both the Ang/Tie 2 and VEGF/VEGFR pathways markedly increased the therapeutic efficacy, therefore may be the preferred approach for treating $\mathrm{RNV}$, in particular ROP.

\section{Acknowledgements}

Not applicable.

\section{Funding}

No funding was received.

\section{Availability of data and materials}

The datasets analyzed during the current study are available from the corresponding author on reasonable request.

\section{Authors' contributions}

WL and YL designed the study and performed the experiments. WZ, CuZ and ChZ established the animal models. WZ and XL collected the data. WL and YZ analyzed the data.
WL and YL prepared the manuscript. All authors read and approved the final manuscript.

\section{Ethics approval and consent to participate}

This study was approved by the Animal Ethics Committee of Liaocheng People's Hospital Animal Center.

\section{Patient consent for publication}

Not applicable.

\section{Competing interests}

The authors declare that they have no competing interests.

\section{References}

1. Kahn HA and Hiller R: Blindness caused by diabetic retinopathy. Am J Ophthalmol 78: 58-67, 1974.

2. Gibson DL, Sheps SB, Uh SH, Schechter MT and McCormick AQ: Retinopathy of prematurity-induced blindness: Birth weight-specific survival and the new epidemic. Pediatrics 86: 405-412, 1990.

3. Jasani B, Nanavati R and Kabra N: Mechanisms and management of retinopathy of prematurity. N Engl J Med 368: 1161-1162, 2013.

4. Rao RC and Dlouhy BJ: Mechanisms and management of retinopathy of prematurity. N Engl J Med 368: 1161, 2013.

5. Hartnett ME and Penn JS: Mechanisms and management of retinopathy of prematurity. N Engl J Med 367: 2515-2526, 2012.

6. Connor KM, Krah NM, Dennison RJ, Aderman CM, Chen J, Guerin KI, Sapieha P, Stahl A, Willett KL and Smith LE: Quantification of oxygen-induced retinopathy in the mouse: A model of vessel loss, vessel regrowth and pathological angiogenesis. Nat Protoc 4: 1565-1573, 2009.

7. Liu Y, Liang X, Xu C, Xie S, Kuang W and Liu Z: Quantification of oxygen-induced retinopathy in the mouse. Yan Ke Xue Bao 22: 103-106, 2006 (In Chinese).

8. Ding X, Liang X, Xie S, Zhu X and Tang S: A modified mouse model of oxygen-induced retinopathy. Yan Ke Xue Bao 22: 98-102, 2006 (In Chinese).

9. Smith LE, Wesolowski E, McLellan A, Kostyk SK, D'Amato R, Sullivan R and D'Amore PA: Oxygen-induced retinopathy in the mouse. Invest Ophthalmol Vis Sci 35: 101-111, 1994.

10. Sato T, Wada K, Arahori H, Kuno N, Imoto K, Iwahashi-Shima C and Kusaka S: Serum concentrations of bevacizumab (avastin) and vascular endothelial growth factor in infants with retinopathy of prematurity. Am J Ophthalmol 153: 327-333.e1, 2012.

11. Käll A: Is Avastin the right choice of treatment for retinopathy of prematurity? Acta Paediatr 101: 796-798, 2012.

12. Avery RL: Bevacizumab (Avastin) for retinopathy of prematurity: Wrong dose, wrong drug, or both? J AAPOS 16: 2-4, 2012.

13. Nuti E, Traversi C, Marigliani D, Balestrazzi A, Alegente M, Martone G, Malandrini A, Romeo N, Mazzotta C and Tosi GM: Treatment of macular edema because of occlusive vasculitis with bevacizumab (avastin): Efficacy of three consecutive monthly injections. Retina 31: 1863-1870, 2011.

14. Wu L, Arevalo JF, Roca JA, Maia M, Berrocal MH, Rodriguez FJ, Evans T, Costa RA and Cardillo J; Pan-American Collaborative Retina Study Group (PACORES): Comparison of two doses of intravitreal bevacizumab (Avastin) for treatment of macular edema secondary to branch retinal vein occlusion: Results from the Pan-American Collaborative Retina Study Group at 6 months of follow-up. Retina 28: 212-219, 2008.

15. Kook D, Wolf A, Kreutzer T, Neubauer A, Strauss R, Ulbig M, Kampik A and Haritoglou C: Long-term effect of intravitreal bevacizumab (avastin) in patients with chronic diffuse diabetic macular edema. Retina 28: 1053-1060, 2008.

16. Wu WC, Kuo HK, Yeh PT, Yang CM, Lai CC and Chen SN: An updated study of the use of bevacizumab in the treatment of patients with prethreshold retinopathy of prematurity in taiwan. Am J Ophthalmol 155: 150-158.e1, 2013. 
17. Wang X, Bullock AJ, Zhang L, Wei L, Yu D, Mahagaokar K, Alsop DC, Mier JW, Atkins MB, Coxon A, et al: The role of angiopoietins as potential therapeutic targets in renal cell carcinoma. Transl Oncol 7: 188-195, 2014.

18. Saulle E, Guerriero R, Petronelli A, Coppotelli E, Gabbianelli M, Morsilli O, Spinello I, Pelosi E, Castelli G, Testa U and Coppola S: Autocrine role of angiopoietins during megakaryocytic differentiation. PLoS One 7: e39796, 2012.

19. Thomas M and Augustin HG: The role of the Angiopoietins in vascular morphogenesis. Angiogenesis 12: 125-137, 2009.

20. Tuo QH, Zeng H, Stinnett A, Yu H, Aschner JL, Liao DF and Chen JX: Critical role of angiopoietins/Tie-2 in hyperglycemic exacerbation of myocardial infarction and impaired angiogenesis. Am J Physiol Heart Circ Physiol 294: H2547-H2557, 2008.

21. Hildbrand P, Cirulli V, Prinsen RC, Smith KA, Torbett BE, Salomon DR and Crisa L: The role of angiopoietins in the development of endothelial cells from cord blood CD34+ progenitors. Blood 104: 2010-2019, 2004.

22. Plank MJ, Sleeman BD and Jones PF: The role of the angiopoietins in tumour angiogenesis. Growth Factors 22: 1-11, 2004

23. Thurston G: Role of Angiopoietins and Tie receptor tyrosine kinases in angiogenesis and lymphangiogenesis. Cell Tissue Res 314: 61-68, 2003

24. Ellis LM, Ahmad S, Fan F, Liu W, Jung YD, Stoeltzing O, Reinmuth $\mathrm{N}$ and Parikh AA: Angiopoietins and their role in colon cancer angiogenesis. Oncology (Williston Park) 16: 31-35, 2002.

25. Campochiaro PA, Sophie R, Pearlman J, Brown DM, Boyer DS Heier JS, Marcus DM, Feiner L and Patel A: Long-term outcomes in patients with retinal vein occlusion treated with ranibizumab: The RETAIN study. Ophthalmology 121: 209-219, 2014.

26. Chung NA, Makin AJ and Lip GY: Measurement of the soluble angiopoietin receptor tie-2 in patients with coronary artery disease: Development and application of an immunoassay. Eur J Clin Invest 33: 529-535, 2003.

27. Wang $\mathrm{C}, \mathrm{Fu} \mathrm{P}, \mathrm{Li} \mathrm{H}$, Gao R and Xiu R: Soluble angiopoietin receptor Tie-2 in patients with acute myocardial infarction and its effects on angiogenesis. Clin Hemorheol Microcirc 33: 1-10, 2005.

28. Singh N, Macnamara E, Rashid S, Ambati J, Kontos CD, Higgins E and Ambati BK: Systemic soluble Tie2 expression inhibits and regresses corneal neovascularization. Biochem Biophys Res Commun 332: 194-199, 2005.

29. Takagi H, Koyama S, Seike H, Oh H, Otani A, Matsumura M and Honda Y: Potential role of the angiopoietin/tie2 system in ischemia-induced retinal neovascularization. Invest Ophthalmol Vis Sci 44: 393-402, 2003.

30. Hangai M, Moon YS, Kitaya N, Chan CK, Wu DY, Peters KG, Ryan SJ and Hinton DR: Systemically expressed soluble Tie2 inhibits intraocular neovascularization. Hum Gene Ther 12 : 1311-1321, 2001.

31. Hohlbaum K, Bert B, Dietze S, Palme R, Fink H and Thone-Reineke $\mathrm{C}$ : Impact of repeated anesthesia with ketamine and xylazine on the well-being of C57BL/6JRj mice. PLoS One 13: e203559, 2018.
32. Shim HJ, Jung WB, Schlegel F, Lee J, Kim S, Lee J and Kim SG: Mouse fMRI under ketamine and xylazine anesthesia: Robust contralateral somatosensory cortex activation in response to forepaw stimulation. Neuroimage 177: 30-44, 2018.

33. Stewart KA, Wilcox KS, Fujinami RS and White HS: Development of postinfection epilepsy after Theiler's virus infection of C57BL/6 mice. J Neuropathol Exp Neurol 69: 1210-1219, 2010.

34. You JY, Chung H and Kim HC: Evaluation of changes in choroidal neovascularization secondary to age-related macular degeneration after anti-VEGF therapy using spectral domain optical coherence tomography. Curr Eye Res 37: 438-445, 2012.

35. Oishi A, Yamashiro K, Tsujikawa A, Ooto S, Tamura H, Nakata I, Miyake $\mathrm{M}$ and Yoshimura N: Long-term effect of intravitreal injection of anti-VEGF agent for visual acuity and chorioretinal atrophy progression in myopic choroidal neovascularization. Graefes Arch Clin Exp Ophthalmol 251: 1-7, 2013.

36. Introini U, Casalino G, Querques G, Gimeno AT, Scotti F and Bandello F: Spectral-domain OCT in anti-VEGF treatment of myopic choroidal neovascularization. Eye (Lond) 26: 976-982, 2012 .

37. Lee J, Park DY, Park DY, Park I, Chang W, Nakaoka Y, Komuro I, Yoo OJ and Koh GY: Angiopoietin-1 suppresses choroidal neovascularization and vascular leakage. Invest Ophthalmol Vis Sci 55: 2191-2199, 2014

38. Wang Y, Bi H, Teng D, Zou Y, Pan X, Guo D and Cui Y: Potential protective effect of angiopoietin-1 on the leakage of rat choroidal neovascularization. Saudi Med J 34: 584-590, 2013.

39. Feng Y, Wang Y, Pfister F, Hillebrands JL, Deutsch U and Hammes HP: Decreased hypoxia-induced neovascularization in angiopoietin-2 heterozygous knockout mouse through reduced MMP activity. Cell Physiol Biochem 23: 277-284, 2009.

40. Natarajan G, Johnson YR, Brozanski B, Farrow KN, Zaniletti I, Padula MA, Asselin JM, Durand DJ, Short BL, Pallotto EK, et al: Postnatal weight gain in preterm infants with severe bronchopulmonary dysplasia. Am J Perinatol 31: 223-230, 2014.

41. Turner S, Zhang G, Young S, Cox M, Goldblatt J, Landau L and Le Souëf P: Associations between postnatal weight gain, change in postnatal pulmonary function, formula feeding and early asthma. Thorax 63: 234-239, 2008.

42. Stahl A, Krohne TU, Eter N, Oberacher-Velten I, Guthoff R, Meltendorf S, Ehrt O, Aisenbrey S, Roider J, Gerding H, et al: Comparing alternative ranibizumab dosages for safety and efficacy in retinopathy of prematurity: A randomized clinical trial. Jama Pediatr 172: 278-286, 2018.

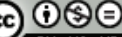

This work is licensed under a Creative Commons Attribution-NonCommercial-NoDerivatives 4.0 International (CC BY-NC-ND 4.0) License. 